\title{
Les facteurs qui influent sur le rendement à une tâche de français chez les femmes d'origine acadienne : évocation de catégories sociales et confiance dans son rendement
}

Josée LeBlanc

Ann M. Beaton 1

Université de Moncton

Résumé

Cette étude examine l'effet de l'évocation de catégories sociales sur le rendement à une tâche en français auprès de femmes d'origine acadienne. Selon la théorie de la menace du stéréotype, l'évocation d'une catégorie sociale entachée d'une mauvaise réputation dans un domaine d'activités perturbe le rendement dans ce domaine. Dans cette étude, 43 participantes ont été placées dans l'une de trois conditions expérimentales, deux évoquant les catégories sociales Femme $(n=15)$ et Acadien $(n=15)$, alors que la troisième, la condition Contrôle $(n=13)$, n'évoquait aucune catégorie. Elles ont ensuite effectué une tâche écrite d'évaluation du rendement en français. Les résultats d'une analyse de covariance révèlent qu'indépendamment de facteurs tels que l'importance accordée au français, la compétence perçue en français, la note obtenue au dernier cours de français et la région d'origine, l'évocation de la catégorie sociale Acadien influence le rendement en français. Les participantes de la condition Acadien ont en effet obtenu un résultat plus faible à la tâche que celles occupant les conditions Femme et Contrôle. Aucune différence significative n'a été obtenue entre ces deux dernières conditions. De plus, selon les résultats, la confiance des participantes dans leur rendement atténue l'effet de l'évocation de la catégorie sociale Acadien sur le rendement en français. Les retombées pratiques sont abordées.

\section{Abstract}

The purpose of this study is to examine the effect of evoking a social category on performance on a French test among women of Acadian heritage. According to the stereotype threat theory, when a social category that contains

\footnotetext{
${ }^{1}$ Prière d'adresser toute correspondance à Ann Beaton, École de psychologie, Université de Moncton ou par courriel à ann.beaton@umoncton.ca.

Ce projet a été financé par une bourse d'études supérieures du Conseil de recherches en sciences humaines du Canada accordée à la première auteure (767-2010-1721) et par une subvention du Conseil de recherches en sciences humaines du Canada (950203481) accordée à la deuxième auteure.
}

Volume 6, numéro 1, 2011 
a negative reputation in a domain is evoked, task performance in this domain will be disrupted. In this study, 43 participants were placed in one of three experimental conditions, two evoking the social categories Woman $(n=15)$ and Acadian ( $n=15)$, the third one, the Control $(n=13)$ condition, evoking no social category. They were subsequently asked to complete a written French test. Results of an analysis of covariance reveal that, over and above the influence of factors such as importance attributed to French, perceived competence in French, performance in the last French class, and place of birth, the evocation of the social category Acadian affects achievement on the French test. Participants in the Acadian condition performed more poorly on the test than participants in the Woman and Control conditions. No significant difference was found between these two last conditions. Furthermore, according to the results, confidence in one's performance reduces the effect of the social category Acadian on test performance. Practical implications are discussed. 


\section{Introduction}

La mobilisation des Acadiennes et des Acadiens pour exiger des mesures visant à conserver et à protéger leur langue a laissé des retombées importantes sur le plan éducatif au Canada (Cormier, 2005; Foucher, 1999). Cependant, malgré ces efforts, la situation linguistique en Acadie demeure précaire. Effectivement, les résultats obtenus aux épreuves nationales et internationales (Groupe DIEPE, 1995; Programme international pour le suivi des acquis des élèves [PISA], 2001, 2003, 2006) révèlent que les jeunes francophones du Nouveau-Brunswick font preuve d'un plus faible rendement en français que les jeunes francophones provenant d'un milieu majoritaire. De plus, des différences se manifestent sur le plan de la compétence langagière selon les régions du Nouveau-Brunswick, bien que les résultats des recherches dans ce domaine sont mitigés: alors que Landry et Allard (1994) ont trouvé que le rendement en français des francophones du sud du Nouveau-Brunswick est plus faible que celui des francophones du nord de cette province, les données du Ministère de l'Éducation du Nouveau-Brunswick (2009) révèlent que les élèves du sud-est du Nouveau-Brunswick sont les plus performants en français de la province.

Les faibles résultats en français obtenus par les jeunes francophones du Nouveau-Brunswick (Groupe DIEPE, 1995; PISA, 2001, 2003, 2006) peuvent s'expliquer de plusieurs façons. Selon le modèle des déterminants du bilinguisme additif et du bilinguisme soustractif de Landry et Allard (1990; voir aussi Allard, Landry et Deveau, 2006), les compétences langagières des membres d'un groupe linguistique sont largement influencées par la vitalité ethnolinguistique de la communauté et par leur vécu sociolangagier. Boudreau et Dubois (1991) soutiennent que le rendement en français des francophones en Acadie est tributaire de leur situation sociolinguistique. Plus précisément, ces chercheures expliquent que certaines régions du Nouveau-Brunswick sont en situation diglossique où la langue anglaise est dominante, tandis que certaines autres régions, comme le sud-est du Nouveau-Brunswick, sont en situation de triglossie, mettant en contact deux langues de prestige, l'anglais et le français standard, et une langue vernaculaire, appelée le chiac. Ce dernier se caractérise par des emprunts lexicaux, morphologiques et syntaxiques à l'anglais et l'usage d'acadianismes. Boudreau (1991) explique que, quoique les jeunes Acadiens accordent de l'importance à leur langue et expriment le désir de s'épanouir en français, ils révèlent également une autodépréciation et un sentiment d'infériorité par rapport à leur capacité de s'exprimer en français standard. Cette évaluation négative des compétences langagières de la part des Acadiens s'exprime dans des situations particulières. Boudreau et Gadet (1998) avancent que ce sentiment d'inadéquation est éprouvé lorsque la personne d'origine 
acadienne est appelée à se servir du français dans des situations formelles, telles que le contexte d'évaluation du rendement langagier. Or, jusqu'à maintenant, les études n'ont pas évalué de façon systématique les caractéristiques du contexte dans lequel l'évaluation se déroule, c'est-à-dire les facteurs qui favorisent ou qui entravent le rendement des Acadiens à une tâche de compétence en français. Le but de la présente étude est d'analyser l'impact de certains de ces facteurs en fonction de la théorie de la menace du stéréotype (MDS) (Steele et Aronson, 1995; Steele, Spencer et Aronson, 2002). Par conséquent, la présente étude offre un nouvel éclairage sur les facteurs spécifiques au contexte d'évaluation qui sont susceptibles d'influencer le rendement des Acadiens aux épreuves de compétence langagière.

\section{Contexte théorique}

Selon Steele et Aronson (1995), la MDS se manifeste lorsqu'une personne effectue une tâche dans un domaine où la réputation de la catégorie sociale à laquelle elle appartient est entachée par des stéréotypes dégradants. Chaque personne peut appartenir à diverses catégories sociales selon la perspective qu'elle envisage : Canadien, Acadien, francophone, anglophone, bilingue, étudiant, femme, etc. Selon Corneille et Leyens (1994), la catégorie sociale est le résultat d'un processus cognitif et elle regroupe tous les individus à qui l'on attribue les mêmes caractéristiques. Bien souvent, les caractéristiques associées à une catégorie sociale sont issues des stéréotypes véhiculés à l'endroit de cet ensemble d'individus. Les stéréotypes correspondent à un « ...contenu évaluatif attribué à un groupe de personnes, ils comprennent aussi et surtout le «pourquoi », l'explication du fait que ces personnes constituent un groupe et l'explication de leurs ressemblances " (Yzerbyt et Schadron, 1994, p. 139). Ainsi, selon la MDS, lorsqu'une personne est appelée à effectuer une tâche dans un domaine où les stéréotypes de la catégorie sociale remettent en cause ses capacités dans ce domaine, sa crainte de confirmer ces stéréotypes ou de se faire juger à partir de ceux-ci accapare ses ressources cognitives et, par ricochet, perturbe son rendement. Autrement dit, la MDS est une expérience ponctuelle et éphémère qui est déclenchée par certaines caractéristiques du contexte dans lequel l'évaluation se déroule, telles que l'évocation d'une catégorie sociale imbue de stéréotypes d'incapacité dans un domaine (Désert, Croizet et Leyens, 2002). Par exemple, des participants africains-américains à qui on a demandé d'identifier leur groupe ethnique juste avant l'administration d'un test d'intelligence ont fait preuve d'un rendement plus faible que ceux à qui l'on n'a pas demandé d'identifier leur groupe (Steele et Aronson, 1995). Les effets délétères de l'activation d'une catégorie sociale qui fait l'objet de stéréotypes négatifs sur le rendement dans un contexte scolaire ont été documentés auprès de quelques groupes minoritaires, notamment les femmes qui effectuent une tâche en mathématiques (Beaton, Tougas, Rinfret, Huard et Delisle, 2007; Inzlicht et Ben-Zeev, 2003) et les minorités ethniques qui exécutent des activités intellectuelles (Steele et Aronson, 1995). De plus, selon Aronson, Lustina, Cook, Keough, Steele et Brown (1999), les effets pernicieux de la MDS se manifestent surtout auprès de gens qui accordent de l'importance au domaine évalué et qui se perçoivent comme étant compétents.

Une catégorie sociale peut être évoquée de deux façons, soit de façon explicite, avec mention ouverte des stéréotypes qui lui sont associés, soit de façon implicite, sans mention des stéréotypes. Ces deux approches produisent des résultats différents sur le rendement à une tâche. Plus précisément, Cheryan et Bodenhausen (2000) ont examiné l'effet de l'évocation explicite de la catégorie sociale sur le rendement à une tâche en mathématiques 
auprès de femmes d'origine asiatique aux États-Unis. Les participantes ont été invitées à réfléchir à la façon dont la catégorie sociale Femme est évaluée dans la société. Cette catégorie sociale, imbue de stéréotypes désavantageux dans le cadre des mathématiques, a donc été évoquée de façon explicite. Les résultats indiquent que cette approche est venue contrecarrer les effets de la MDS. II semble que l'évocation explicite de l'image stéréotypée désavantageuse n'a pas provoqué une baisse de performance, les participantes s'étant par la suite davantage appliquées à la tâche (Cheryan et Bodenhausen, 2000). La réussite dans une tâche exécutée par les membres d'un groupe stigmatisé, lorsque les stéréotypes avilissants sont manifestes, a également été démontrée dans une étude menée par Oswald et Harvey (2000-2001). Par contre, les recherches indiquent que lorsque la catégorie sociale est évoquée de façon plus subtile ou implicite, voire tacite, les effets pernicieux de la MDS sur le rendement sont davantage susceptibles de se manifester (Steele, 1997; Shih, Ambady, Richeson, Fujita et Gray, 2002; Shih, Pittinsky et Ambady, 1999). Cela est d'un intérêt particulier dans le contexte scolaire car, comme le soulignent Inzlicht, Aronson et Mendoza-Denton (2009), ce sont surtout des facteurs subtils qui sont en jeu dans un tel contexte et qui ont comme effet d'évoquer la catégorie sociale d'une manière implicite.

Certaines recherches sur la MDS visent aussi à cerner d'autres facteurs qui pourraient contrecarrer les effets pernicieux de la MDS, tels que le choix de la catégorie sociale évoquée et le niveau de confiance des individus en leurs capacités. En ce qui concerne le choix de la catégorie sociale, la recherche de Shih, Pittinsky et Ambady (1999) suggère que ce ne sont pas toutes les catégories sociales qui suscitent une baisse de performance. Ces auteurs ont examiné les effets de l'activation de diverses catégories sociales caractérisées par différentes images stéréotypées par rapport au rendement dans un domaine précis. Dans une première étude, les auteurs ont examiné le rendement d'étudiantes d'origine asiatique à une épreuve de mathématiques aux États-Unis. Ces participantes occupent plusieurs catégories sociales, dont celles de Femme et d'Asiatique. Ces deux catégories sociales se caractérisent par différents stéréotypes en rapport aux capacités dans le domaine des mathématiques. Plus précisément, selon Shih, Pittinsky et Ambady (1999), bien que la catégorie sociale Femme évoque des stéréotypes négatifs qui mettent en doute la compétence en mathématiques, la catégorie sociale Asiatique est plutôt associée à une image positive de compétence dans les domaines quantitatifs. Tel que postulé, lorsque les chercheurs ont évoqué la catégorie sociale Femme, le rendement des participantes au test de mathématiques s'est avéré inférieur à celui des participantes de la condition expérimentale Contrôle, et à celui des participantes de la condition où la catégorie sociale Asiatique a été rendue saillante. Ainsi, l'évocation de la catégorie sociale peut augmenter ou diminuer le rendement à une tâche, dépendant des stéréotypes positifs ou négatifs qui s'y rattachent (voir également les travaux de Harrison, Stone, Shapiro, Yee, Boyd et Rullan, 2009; Yopyk et Prentice, 2005). Cependant, dans une deuxième étude semblable menée par Shih, Pittinsky et Ambady (1999), auprès de femmes d'origine asiatique au Canada, ces résultats ne se sont pas reproduits. Par conséquent, les effets de l'évocation de la catégorie sociale Femme et de l'évocation d'une catégorie sociale associée à un groupe culturel sur le rendement à une tâche apparaissent mitigés. II est possible que les stéréotypes qui leur sont associés varient d'un contexte à l'autre.

En ce qui concerne le niveau de confiance dans son rendement, l'influence de ce facteur dans le contexte de la MDS demeure obscur (Smith, 2004; Steele, Spencer et Aronson, 2002). Les études ayant évalué l'impact du sentiment de confiance avant (Cadinu, Maass, Frigerio, Impagliazzo et Latinotti, 2003; Sekaquaptewa et Thompson, 
2003) ou suivant (Aronson, Lustina, Gook, Keough, Steele et Brown, 1999; Keller, 2002; Shih, Pittinsky et Ambady, 1999) une tâche dans une condition de la MDS révèlent des effets mitigés. Plus précisément, lorsque la confiance dans son rendement est mesurée après l'exécution de la tâche, ce facteur ne semble pas moduler le rendement en tant que tel. Cependant, lorsque la confiance dans son rendement est mesurée avant l'activité, on constate un effet modeste sur le rendement (Cadinu, Maass, Frigerio, Impagliazzo et Latinotti, 2003). Puisque la MDS est un phénomène ponctuel (Steele et Aronson, 1995), l'évaluation de la confiance dans son rendement pendant l'exécution de la tâche pourrait apporter un nouvel éclairage sur ces résultats mixtes et aider à mieux cerner les effets de ce facteur dans le contexte de la MDS.

En somme, les études antérieures démontrent que l'évocation implicite d'une catégorie sociale entachée d'un stéréotype positif ou négatif peut influencer le rendement à une tâche dans un domaine qui se rapporte au stéréotype (Shih, Pittinsky et Ambady, 1999; Steele et Aronson, 1995). Lorsque la catégorie sociale évoquée est associée à un stéréotype négatif, on observe une diminution du rendement. Lorsque la catégorie sociale est associée à un stéréotype positif, on observe une meilleure performance à la tâche. De plus, il y a raison de croire que le niveau de confiance dans son rendement pourrait également moduler le rendement à une tâche, dans la mesure où ce niveau de confiance est évalué dans le cours même de la réalisation de la tâche.

\section{Position du problème, objectifs et hypothèses de la recherche}

L'étude de l'influence de la MDS sur le rendement dans une tâche de français, tout autant que l'examen de la confiance dans son rendement pendant la réalisation d'une telle tâche, apparaissent particulièrement appropriés pour la population ciblée dans cette recherche, les femmes d'origine acadienne. En effet, il existe un stéréotype positif par rapport à la catégorie sociale Femme et un stéréotype négatif par rapport à la catégorie sociale Acadien quant au rendement en français. De plus, on sait que l'insécurité linguistique, qui correspond à la conviction que son discours langagier est inadéquat et que sa capacité à s'exprimer dans une langue juste et correcte est inatteignable (Bourdieu, 1982; Cormier, 2005; Labov, 1976), est un enjeu important chez les Acadiens. Plus précisément, l'insécurité linguistique risque de porter atteinte à leur rendement langagier (Boudreau et Dubois, 1991, 1992).

Ainsi, la présente étude se fixe deux objectifs principaux. Dans un premier temps, elle vise à examiner les effets de l'évocation de catégories sociales sur le rendement à une tâche de français chez des femmes d'origine acadienne. Il est postulé que le rendement à une tâche en français est modulé selon les catégories sociales Femme et Acadien. Plus précisément, par rapport à leur compétence langagière, les femmes d'origine acadienne sont la cible à la fois d'un stéréotype positif ("Les femmes sont bonnes en français », Inzlicht et Ben-Zeev, 2000) et d'un stéréotype négatif ("Les Acadiens ne sont pas bons en français », Boudreau et Dubois, 2001). Les hypothèses stipulent que les participantes pour qui la catégorie sociale Acadien sera évoquée, manifesteront un rendement plus faible à une tâche de français que celles pour qui la catégorie sociale Femme, et celles pour qui aucune catégorie sociale particulière (condition Contrôle) par rapport au rendement en français seront évoquées. De plus, il est postulé que les participantes pour qui la catégorie sociale Femme sera évoquée auront une meilleure performance en français que celles occupant la condition Contrôle. L'examen de l'effet de l'évocation de différentes catégories sociales sur le rendement à une tâche en français est susceptible d'offrir des pistes de réflexion aux éducatrices et aux éducateurs 
qui souhaitent créer un milieu favorisant la réussite scolaire des jeunes francophones en situation minoritaire (Cormier, 2005; Cormier, Pruneau, Rivard et Blain, 2004).

Le deuxième objectif de cette étude est d'évaluer le rôle de la confiance en ses capacités lors d'une tâche effectuée dans le contexte de la MDS. Puisque la MDS est un phénomène ponctuel (Steele et Aronson, 1995), il est postulé que l'auto-évaluation de la confiance dans ses propres compétences réalisée tout au long de l'exécution de la tâche aura comme conséquence de protéger l'individu contre les effets pernicieux de la MDS.

\section{Méthodologie}

\section{Participantes}

En tout, 43 femmes d'origine acadienne ont participé à cette étude. Les participantes, âgées en moyenne de 20,6 ans, étaient inscrites au premier cycle d'un établissement universitaire francophone. La majorité d'entre elles $(60,9 \%)$ suivaient un programme d'études de la Faculté des sciences de la santé et des services communautaires. Les participantes provenaient du nord $(50 \%)$ et du sud $(43,5 \%)$ du Nouveau-Brunswick ainsi que d'autres régions de l'est du Canada (6,5\%). La plupart des répondantes (91,3\%) ont suivi au moins un cours universitaire en français, en linguistique ou en littérature française.

\section{Procédure expérimentale}

Ce projet de recherche s'est réalisé en deux étapes. Au cours de la première étape, un questionnaire a été distribué dans le cadre de différents cours du premier cycle. En plus d'identifier le sexe et les coordonnées des étudiantes et étudiants, ce questionnaire visait à recueillir l'information concernant les covariables d'intérêt pour cette étude. Ainsi, le sentiment de compétence en français et l'importance accordée à ce domaine ont été mesurés à partir des énoncés suivants : Je suis bon, bonne en français et C'est important pour moi d'être bon, bonne en français. Les étudiants ont exprimé leurs réactions sur une échelle de type Likert de 7 points, où 1 correspond à absolument en désaccord et 7 , à absolument d'accord. Le rendement au dernier cours de français a également été évalué selon l'échelle suivante : $1=$ entre $A+$ et $A-, 2=$ entre $B+$ et $B$-, $3=$ entre $C+$ et $C$-, $4=$ entre $D+$ et $D$ - et $5=$ Échec. Enfin, pour la covariable région d'origine (nord du Nouveau-Brunswick, sud du Nouveau-Brunswick et est du Canada) les étudiants ont indiqué leur ville natale. Seuls les questionnaires remplis par les femmes d'origine acadienne ont été retenus et ces dernières ont été invitées à participer à la deuxième étape du projet en laboratoire.

Les participantes ont été assignées de façon aléatoire aux trois conditions expérimentales suivantes: Femme $(n=15)$, Acadien $(n=15)$ et Contrôle $(n=13)$. Une assistante de recherche qui ne connaissait ni les hypothèses, ni les conditions expérimentales a accueilli des groupes de 8 à 10 participantes au laboratoire afin d'effectuer deux tâches. Comme première tâche, les participantes ont rempli un formulaire intitulé Sondage sur la vie des étudiants. Cette tâche, qui s'inspire de la méthodologie de Shih, Pittinsky et Ambady (1999), visait à évoquer les catégories sociales Femme ou Acadien, ou à procurer une condition Contrôle n'évoquant aucune catégorie sociale. Conformément à la technique utilisée par ces auteurs, la catégorie sociale Femme a été évoquée à l'aide de six questions : 1. Tu es : a) une femme, b) un homme? 2. Habites-tu en résidence à (nom de l'université)? 3. As-tu un ou une colocataire? 4. Que préfères-tu : une résidence mixte ou une résidence pour un sexe seulement? 5 . Énumère 3 
avantages de vivre en résidence mixte. 6. Énumère 3 avantages de vivre en résidence pour un sexe seulement. Afin d'évoquer la catégorie sociale Acadien, les six questions suivantes ont été posées: 1. Qui es-tu: a) Acadien, b) Québécois, c) Autre? 2. Par rapport au groupe que tu as identifié à la question précédente, énumère 3 caractéristiques de ton groupe. 3. Quelle est la ville natale de tes parents et de tes grands-parents? 4. Quelle est ta ville natale? 5. Astu célébré le 15 août cette année? Si oui, comment l'as-tu célébré? 6. Connais-tu la télésérie AcadieMan? Si oui, que penses-tu de cette émission? II est à noter que le 15 août est la fête nationale des Acadiens et « AcadieMan » est la première télésérie où figure «Le First Superhero acadien ». Toujours en suivant l'approche de Shih, Pittinsky et Ambady (1999), la condition Contrôle a été établie grâce à six questions portant sur les services offerts à l'université : 1. Habites-tu en résidence à (nom de l'université)? 2. Utilises-tu ton courriel de (nom de l'université)? 3. Est-ce que tu es satisfait du service de courriel offert par (nom de l'université)? 4. Es-tu abonné au câble de télévision? 5 . Énumère 3 avantages de l'accès au câble de télévision. 6. Énumère 3 désavantages de l'accès au câble de télévision.

À la suite du Sondage sur la vie des étudiants, les participantes devaient répondre à un test visant à évaluer leurs compétences en français. Ce test contenait 29 questions à choix multiples portant sur l'orthographe (p. ex. Indiquez lequel des mots suivants est mal orthographié : a) rénumérer, b) exercice, c) infarctus), la syntaxe (p. ex. Quelle formulation parmi les suivantes est fautive? a) La fille que je sors avec, b) La fille avec qui je sors, c) La fille avec laquelle je sors) et la conjugaison de verbes (p. ex. Fontaine, je ne plus de ton eau. a) buverai, b) boirai, c) boierai). Les questions ont été tirées du site Internet du Centre collégial de développement de matériel didactique, un centre de production de matériel pédagogique à l'intention du personnel enseignant et des étudiants du réseau collégial du Québec ( $\underline{w w w . c c d m d . q c . c a})$. Les participantes ont été informées qu'il s'agissait d'une épreuve communément utilisée dans le contexte scolaire afin d'évaluer le rendement en français.

Dans le but d'évaluer la confiance des participantes dans leur rendement tout au long du test de français, l'énoncé suivant a été reproduit après chacune des questions : Par rapport à cette question à choix multiple, à quel point es-tu certaine que ta réponse est juste? Encercle le chiffre qui correspond à ton opinion. Les réponses ont été recueillies sur une échelle de type Likert de 7 points, où 1 correspond à complètement incertaine et 7 , à complètement certaine.

À la fin de ces tâches, l'expérimentatrice a vérifié la présence de soupçons chez les participantes concernant la manipulation expérimentale et leur a fourni l'occasion de poser leurs questions. Avant de quitter le laboratoire, l'expérimentatrice a dévoilé le but véritable de l'étude et a remercié les répondantes de leur participation à la recherche.

\section{Méthodes d'analyse}

Étant donné que le test visant à évaluer les compétences en français n'a pas fait l'objet de validation empirique avant d'être administré aux participantes, il convient tout d'abord de s'assurer qu'il possède des qualités métriques acceptables. En particulier, une analyse du coefficient de difficulté de chacune des questions est effectuée (Anastasi, 1994). Cette analyse vise à repérer les questions trop faciles ou trop difficiles, et à ne conserver que les questions pertinentes dans les analyses statistiques subséquentes. Le calcul du coefficient de consistance interne alpha de Cronbach permet aussi de vérifier la fiabilité de l'épreuve. 
Par la suite, deux analyses de covariance sont effectuées (Tabachnick et Fidell, 2007). La première permet de vérifier si le rendement à l'épreuve de compétence en français varie d'une manière significative selon les trois conditions expérimentales (Femme, Acadien, Contrôle) tout en contrôlant l'effet de quatre facteurs associés au rendement en français : l'importance accordée au français, la compétence perçue en français, le rendement au dernier cours de français et la région d'origine. La deuxième analyse de covariance permet de vérifier si l'effet des conditions expérimentales se maintient ou s'estompe lorsqu'on ajoute la covariable "confiance dans son rendement». Essentiellement, la deuxième analyse vise à déterminer si la confiance dans son rendement est un facteur de protection contre les effets pervers de la MDS. Dans les cas où les analyses de covariance révèlent une différence significative, les analyses a posteriori sont effectuées à l'aide du test LSD de Fisher.

Enfin, pour répondre aux préoccupations concernant la taille de l'échantillon sur la puissance des tests statistiques, le logiciel PASS11 (Power Analysis and Sample Size; http://www.ncss.com/pass.html) a été utilisé. Pour une analyse effectuée auprès de 43 participantes ( $\left.n_{1}=15, n_{2}=15, n_{3}=13\right)$, avec quatre covariables et un seuil de probabilité de 0,05 , la puissance statistique est de $0,84(d=0,48)$, ce qui peut être considéré comme satisfaisant.

\section{Résultats}

\section{Qualités métriques de l'épreuve de compétence en français}

Le calcul du coefficient de difficulté pour chacune des questions du test de compétence en français révèle que 11 questions sur les 29 composant l'épreuve originale s'avèrent trop faciles (plus de $85 \%$ de réussites) ou trop difficiles (moins de $30 \%$ de réussites). Ces énoncés ne rencontrent pas le critère de validité proposé par Anastasi (1994), à savoir que le niveau de difficulté des questions dans une telle épreuve devrait varier modérément autour de $50 \%$. Ces 11 questions ont donc été écartées. Ainsi, le score global rendant compte du niveau de réussite des participantes à l'épreuve de français a été calculé en considérant uniquement les 18 questions dont le coefficient de difficulté se situait entre 30 et $85 \%$. Une analyse de la cohérence interne pour ces 18 questions laisse voir un degré de fiabilité acceptable, la valeur du coefficient alpha de Cronbach se situant à 0,86 . Signalons que le score représentant le niveau de confiance des participantes tout au long de l'épreuve a été calculé lui aussi seulement à partir des réponses fournies aux 18 questions retenues.

\section{Effet des conditions expérimentales sur le rendement au test de compétence en français}

Comme l'indique la lecture du tableau 1, les scores moyens obtenus au test de compétence en français par les participantes aux trois conditions expérimentales s'élèvent à 8,87 pour la condition Femme (s : 2,61), à 7,47 pour la condition Acadien (s : 1,96) et à 9,08 pour la condition Contrôle $(s: 2,10)$. 
Tableau 1

Statistiques descriptives : nombre de participantes $(n)$, moyennes $(M)$ et écart-types $(s)$ pour le rendement en français et les quatre covariables

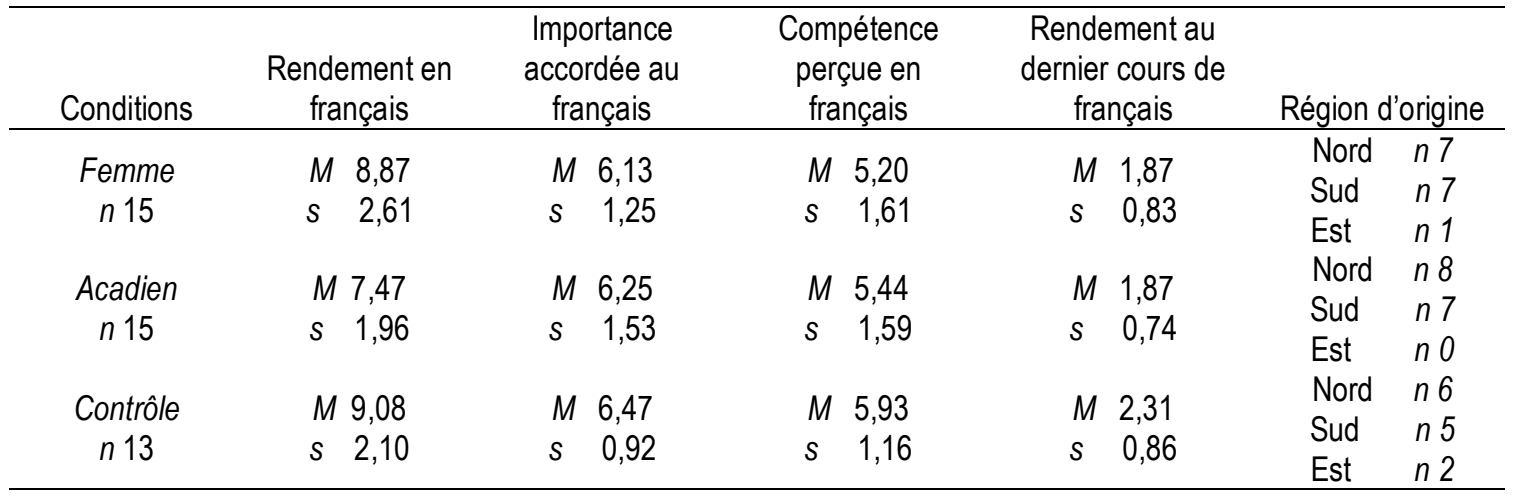

La première analyse de covariance a été menée pour évaluer l'effet des conditions expérimentales (Femme, Acadien, Contrôle) sur le rendement à l'épreuve de compétence en français. L'importance accordée au français, la compétence perçue en français, le rendement au dernier cours de français ainsi que la région d'origine (nord du Nouveau-Brunswick, sud du Nouveau-Brunswick, est du Canada) ont servi de covariables pour cette analyse (voir tableau 1 pour les statistiques descriptives concernant ces quatre covariables). Comme l'indiquent les résultats présentés au tableau 2, l'effet des conditions expérimentales sur le rendement à l'épreuve de compétence en français s'avère significatif $(F[2,36]=3,69, p<, 05)$. L'analyse a posteriori (LSD de Fisher) indique que les participantes de la

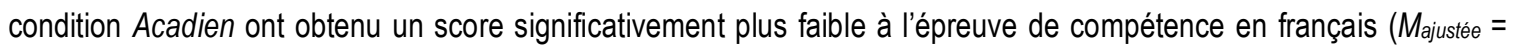
$7,30)$ que les participantes des conditions Femme $\left(M_{\text {ajustée }}=8,95\right)$ et Contrôle $\left(M_{\text {ajustée }}=9,18\right)$. Ce résultat appuie notre première hypothèse : Iorsque la catégorie sociale Acadien est évoquée avant d'effectuer une tâche d'évaluation de la compétence en français, les participantes obtiennent un résultat inférieur comparativement aux participantes de la condition où la catégorie sociale Femme a été rendue saillante et à celles de la condition Contrôle.

Tableau 2

Résultats de la première analyse de covariance

\begin{tabular}{cccc}
\hline Conditions & Moyennes ajustées & $F$ & $p$ \\
\hline Femme & $8,95^{\mathrm{a}}$ & 3,69 &, 03 \\
Acadien & $7,30^{\mathrm{b}}$ & & \\
Contrôle & $9,18^{\mathrm{b}}$ & & \\
\hline
\end{tabular}

Note. Les moyennes marquées ${ }^{a}$ et ${ }^{b}$ diffèrent significativement selon l'analyse a posteriori (LSD de Fisher), $p<, 05$.

Par contre, en ce qui a trait à notre deuxième hypothèse, à savoir que l'évocation de la catégorie sociale Femme devrait avoir un effet positif sur le rendement des participantes assignées à cette condition, comparativement à celles de la condition Contrôle, aucune différence significative n'a été obtenue $(p=, 77)$. Contrairement à nos attentes, les participantes qui occupaient la condition Femme et la condition Contrôle ont fait preuve d'un rendement semblable. II est possible que les questions posées aux participantes de la condition Contrôle n'aient pas été aussi neutres qu'elles le paraissaient à prime abord et aient évoqué une catégorie sociale que nous pourrions appeler Étudiant universitaire, dont les stéréotypes se rapportant à la compétence en français seraient tout aussi positifs que ceux 
associés à la catégorie sociale Femme. Des études plus approfondies seraient toutefois nécessaires pour élucider cette question.

\section{Rôle de la confiance dans son rendement}

Afin d'évaluer le rôle de la confiance des participantes dans leur rendement à la tâche, une deuxième analyse de covariance a été effectuée (voir tableau 3). Conformément à notre troisième hypothèse, l'ajout de cette covariable a fait en sorte que l'effet des conditions expérimentales (Femme, Acadien, Contrôle), s'est estompé ( $F$ [2, $34]=2,91, p>, 05)$. En d'autres termes, tenir compte du niveau de confiance dans son rendement en cours d'exécution de la tâche a eu comme effet d'annuler les différences au niveau du rendement chez les participantes occupant les conditions Femme, Acadien et Contrôle. Ce résultat suggère que la confiance dans son rendement est susceptible de protéger les Acadiennes contre les effets de la MDS lors d'une tâche d'évaluation.

Tableau 3

Résultats de la deuxième analyse de covariance

\begin{tabular}{lccc}
\hline \multicolumn{1}{c}{ Conditions } & Moyennes ajustées & $F$ & $p$ \\
\hline Femme & 8,89 & 2,91 &, 07 \\
Acadien & 7,39 & & \\
Contrôle & 9,15 & & \\
\hline
\end{tabular}

\section{Discussion}

Cette étude a examiné l'effet de l'évocation de catégories sociales sur le rendement de femmes d'origine acadienne à une tâche de compétence en français. Les résultats montrent que lorsque la catégorie sociale Acadien est évoquée, les participantes manifestent une baisse de performance comparativement aux participantes occupant les conditions Femme et Contrôle. Ce résultat appuie la perspective de la MDS et suggère que l'évocation de la catégorie sociale Acadien a comme effet de maintenir et de protéger le stéréotype avilissant à l'endroit du rendement en français de ce groupe minoritaire. Cette étude avait également comme objectif d'évaluer le rôle du niveau de confiance dans son rendement sur les effets pervers de la MDS lors de la réalisation d'une tâche en français. Le résultat de notre analyse indique que ce facteur est susceptible de contrecarrer l'impact de la MDS. Tout en confirmant l'existence d'un facteur particulier qui rompt le lien entre la MDS et la baisse de performance (Wheeler et Petty, 2001), ce résultat contribue à mieux comprendre la place qu'occupe la confiance dans ses compétences au sein de la théorie de la MDS (Smith, 2004; Steele, Spencer et Aronson, 2002).

Par conséquent, la présente étude révèle que la catégorie sociale évoquée dans un contexte de la réalisation d'une tâche d'évaluation de la compétence langagière est suffisante pour faire fluctuer le rendement des participantes. Si, en effet, la saillance de la catégorie sociale Acadien est un facteur qui perturbe le rendement à une tâche en français, ce résultat a des retombées importantes pour l'éducation des jeunes évoluant dans un contexte minoritaire francophone. Plus précisément, les éducateurs auront à prêter davantage attention aux facteurs en jeu dans le contexte scolaire qui soutiennent le lien entre la catégorie sociale Acadien et le stéréotype qui entache sa réputation face aux tâches de français. Une approche pédagogique adaptée à la spécificité culturelle et linguistique 
acadienne, et qui mise sur l'exploitation des auteurs d'origine acadienne se présente, entre autres, comme un moyen dont les enseignants disposent afin de revoir l'image associée aux Acadiens et enrayer la propagation des stéréotypes négatifs.

La présente étude suggère également que les individus ont les moyens de bloquer les effets pernicieux de la MDS. En effet, la confiance dans son rendement a atténué les effets de l'évocation de la catégorie sociale Acadien sur ce rendement. Ainsi, développer des stratégies pour augmenter la confiance des jeunes Acadiens dans leur rendement en français apparaît comme une nécessité. En effet, la confiance en ses capacités en français n'affecte pas seulement le rendement à une tâche ponctuelle. En particulier, l'insécurité linguistique entraîne une baisse de l'estime de soi, de l'attachement au groupe langagier, une moins grande propension à s'exprimer en français, une augmentation de l'assimilation à l'anglais, sans oublier les retombées importantes sur le cheminement scolaire de l'individu (Cormier, Pruneau, Rivard et Blain, 2004). Afin de diminuer l'insécurité linguistique et ses séquelles, une pédagogie adaptée au milieu minoritaire (Boudreau et Dubois, 1991; Cormier, 2005; Cormier, Pruneau, Rivard et Blain, 2004; Francard, 1989), qui reconnaît et valorise les variétés linguistiques et construit sur elles, s'impose. Steele (1997) abonde également en ce sens à la suite d'une intervention visant à favoriser la réussite scolaire des jeunes Africains-Américains, un groupe particulièrement vulnérable aux effets de la MDS en éducation. Parmi les stratégies privilégiées, cet auteur préconise la mise en valeur du potentiel des élèves à atteindre un haut niveau de compétence, tout en assurant que leur rendement ne fera pas l'objet de dénigrement. En d'autres termes, des chercheurs provenant de différents domaines reconnaissent l'importance de créer un climat d'apprentissage sécurisant et valorisant pour les jeunes issus des groupes minoritaires à risque dans un contexte scolaire. II est possible que de telles initiatives dans le contexte scolaire se révèlent bénéfiques pour les individus qui occupent d'autres catégories sociales entachées d'une réputation négative sur le plan langagier, dont les hommes d'origine acadienne.

Par ailleurs, contrairement aux attentes, l'évocation de la catégorie sociale Femme dans la présente étude n'a pas provoqué de rendement supérieur à celui retrouvé dans la condition Contrôle. II est possible que les questions évoquant la condition Contrôle aient activé une catégorie sociale, soit celle d'un individu qui fréquente un établissement universitaire. Est-ce que cette catégorie sociale est associée à une compétence langagière au même titre que la catégorie sociale Femme? Pour le moment, cette question reste ouverte.

Enfin, la présente étude comporte des limites qui doivent être soulignées. D'une part, la tâche de rendement en français a été élaborée pour les fins de cette étude et malgré les efforts de validation déployés, il n'en reste pas moins qu'il n'est pas clair si la tâche est convenable et appropriée. D'autre part, les résultats ont été obtenus en laboratoire, auprès d'un groupe restreint d'étudiantes qui fréquentent un établissement postsecondaire, ce qui appelle une grande prudence dans la généralisation des résultats. Quoique la recherche de Huguet et Régner (2007) a validé les effets pernicieux de la MDS sur le rendement en mathématiques de jeunes filles de $6^{\mathrm{e}}$ et $7^{\mathrm{e}}$ années, à ce jour, aucune validation empirique auprès de jeunes francophones fréquentant les écoles dans un contexte linguistique minoritaire n'a été effectuée. Signalons enfin que la présente étude n'a pas évalué l'attachement des participantes au groupe des Acadiens. Le niveau d'attachement au groupe culturel pourrait s'avérer un autre facteur déterminant sur le rendement à une tâche en français dans le contexte de la MDS. 


\section{Conclusion}

Cette étude révèle que les facteurs en jeu dans le contexte d'évaluation peuvent influencer le rendement des femmes d'origine acadienne à une tâche de français. En particulier, l'évocation de la catégorie sociale Acadien est susceptible d'exercer un effet à la baisse sur le rendement en français, comparativement à l'évocation de la catégorie sociale Femme ou à une condition qui n'évoque aucune catégorie sociale. De plus, cet effet s'estompe lorsque le niveau de confiance dans son rendement à la tâche de français, évalué en cours de réalisation de celle-ci, est considéré. À notre connaissance, il s'agit de la première étude qui démontre les effets de l'évocation de catégories sociales et de la confiance dans son rendement sur la réussite à une épreuve de français chez des femmes appartenant à un groupe francophone minoritaire.

Si les résultats de cette recherche doivent être interprétés avec prudence, ils ouvrent néanmoins la porte à un domaine d'études encore largement inexploré. En particulier, la question à savoir si les effets de l'évocation de la catégorie sociale auprès de femmes francophones minoritaires varient selon différentes modalités d'évaluation reste ouverte. Par exemple, l'évocation de la catégorie sociale Acadien produit-elle les mêmes effets lorsque la tâche évalue les compétences orales en français? Et qu'en est-il de l'effet des variations dans les stéréotypes associés au sexe des participants? La réponse aux questions de ce genre permettrait de mieux cerner les caractéristiques d'une épreuve qui rendent les étudiants davantage à risque de subir les effets pernicieux de la MDS. Également, il s'avérerait important d'effectuer des recherches longitudinales auprès des populations estudiantines afin de mieux comprendre les effets à long terme de la MDS sur leur motivation à maintenir la langue et la culture francophones en milieu minoritaire. Les études dans le domaine de la MDS apparaissent prometteuses pour élargir la compréhension des facteurs qui agissent sur la vitalité ethnolinguistique d'une communauté et éventuellement lutter contre les stéréotypes qui nuisent à sa croissance.

\section{Références}

Allard, R., Landry, R. et Deveau, K. (2006). La vitalité ethnolinguistique et l'étude du développement bilingue des minorités acadiennes et francophones du Canada atlantique. Dans A. Magord (Dir.), Innovation et adaptation : expériences acadiennes contemporaines (p. 85-104). Bruxelles : P.I.E - Peter Lang.

Anastasi, A. (1994). Introduction à la psychométrie. Montréal : Guérin.

Aronson, J. (2002). Stereotype threat : Contending and coping with unnerving expectations. Dans J. Aronson et D. Cordova (Dir.), Improving education : Classic and contemporary lessons from psychology (p. 279-301). New York : Academic Press.

Aronson, J., Lustina, M. J., Gook, C., Keough, K., Steele, C. M. et Brown, J. (1999).When white men can't do math : Necessary and sufficient factors in stereotype threat. Journal of Experimental Social Psychology, 35(1), 29-46.

Beaton, A. M., Tougas, F., Rinfret, N., Huard, N. et Delisle, M.-N. (2007). Strength in numbers? Women and mathematics. European Journal of Psychology of Education, 22(3), 291-306.

Boudreau, A. (1991). Les rapports que de jeunes Acadiens et Acadiennes entretiennent avec leur langue et avec la langue. Égalité, 30, 17-37.

Boudreau, A. et Dubois, L. (1991). L'insécurité linguistique comme entrave à l'apprentissage du français. Revue de l'Association canadienne de linguistique appliquée, 13(2), 37-50.

Boudreau, A. et Dubois, L. (1992). Insécurité linguistique et diglossie : étude comparative de deux régions de l'Acadie du Nouveau-Brunswick. Revue de l'Université de Moncton, 25(1/2), 3-22.

Boudreau, A. et Dubois, L. (2001). Langues minoritaires et espaces publics : le cas de l'Acadie du Nouveau-Brunswick. Estudios de Sociolinguistica, 2(1), 37-60.

Boudreau, A. et Gadet, F. (1998). Attitudes en situation minoritaire. L'exemple de l'Acadie. Dans A. Queffélec (Dir.), Le français en Afrique, francophonies, recueil d'études offert en hommage à Suzanne Lafage (p. 55-61). Nice : Didier Érudition. 
Bourdieu, P. (1982). Ce que parler veut dire : l'économie des échanges linguistiques. Paris : Fayard.

Cadinu, M., Maass, A., Frigerio, S., Impagliazzo, L. et Latinotti, S. (2003). Stereotype threat: The effect of expectancy on performance. European Journal of Social Psychology, 33(2), 267-285.

Cheryan, S. et Bodenhausen, G.V. (2000). When positive stereotypes threaten intellectual performance : The psychological hazards of "model minority" status. Psychological Science, 11(5), 399-402.

Cormier, M. (2005). La pédagogie en milieu minoritaire francophone : une recension des écrits. [En ligne] Disponible le 16 novembre $2010:$ http://icrml.ca/images/stories/documents/fr/cormier_marianne_recension.pdf

Cormier, C., Pruneau, D., Rivard, L. et Blain, S. (2004). Un modèle pédagogique pour améliorer l'apprentissage des sciences en milieu linguistique minoritaire. Francophonies d'Amérique, 18(1), 21-35.

Corneille, O. et Leyens, J.-P. (1994). Catégories, catégorisation sociale et essentialisme psychologique. Dans R. Y. Bourhis et J.-P. Leyens (Dir.), Stéréotypes, discrimination et relations intergroupes (p. 41-68). Liège : Mardaga.

Désert, M., Croizet, J.-C. et Leyens, J.-P. (2002). La menace du stéréotype : une interaction entre situation et identité. L'Année psychologique, 102(3), 555-576.

Foucher, P. (1999). Les droits linguistiques au Canada. Dans J.-Y. Thériault (Dir.), Francophonies minoritaires au Canada : l'état des lieux (p. 307-324). Moncton : Éditions d'Acadie.

Francard, M. (1989). Insécurité linguistique en situation de diglossie : le cas de l'Ardenne belge. Revue québécoise de linguistique théorique et appliquée, 8(2), 133-163.

Groupe DIEPE (1995). Savoir écrire au secondaire : étude comparative auprès de quatre populations francophones d'Europe et d'Amérique. Bruxelles : De Boeck Université.

Harrison, C. K., Stone, J., Shapiro, J., Yee, S., Boyd, J. A. et Rullan, V. (2009). The role of gender identities and stereotype salience with the academic performance of male and female college athletes. Journal of Sport and Social Issues, 33(1), 7896.

Huguet, P. et Régner, I. (2007). Stereotype threat among schoolgirls in quasi-ordinary classroom circumstances. Journal of Educational Psychology, 99(3), 545-560.

Inzlicht, M., Aronson, J. et Mendoza-Denton, R. (2009). On being the target of prejudice : Educational implications. Dans F. Butera et J. M. Levine (Dir.), Coping with minority status: Responses to exclusion and inclusion (p. 13-37). New York: Cambridge University Press.

Inzlicht, M. et Ben-Zeev, T. (2000). A threatening intellectual environment : Why females are susceptible to experiencing problem-solving deficits in the presence of males. Psychological Science, 11(5), 365-371.

Inzlicht, M. et Ben-Zeev, T. (2003). Do high-achievement female students underperform in private? The implications of threatening environments on intellectual processing. Journal of Educational Psychology, 95(4), 796-805.

Keller, J. (2002). Blatant stereotype threat and women's math performance : Self-handicapping as a strategic means to cope with obtrusive negative performance expectations. Sex Roles, 47(3/4), 193-198.

Labov, W. (1976). Sociolinguistique. Paris : Les Éditions de Minuit.

Landry, R. et Allard, R. (1990). Contact des langues et développement bilingue : un modèle macroscopique. La Revue canadienne des langues vivantes/The Canadian Modern Language Review, 46(3), 527-553.

Landry, R. et Allard, R. (1994). The Acadians of New Brunswick : Demolinguistic realities and the vitality of the French language. International Journal of the Sociology of Language, 105/106(1), 181-215.

Ministère de l'Éducation du Nouveau-Brunswick (2009). Résultats des examens : districts scolaires francophones. [En ligne] Disponible le 16 novembre 2010 : http://www.gnb.ca/0000/ publications/evalf/Rapportpublic2009FR.pdf

Oswald, D. L. et Harvey, R. D. (2000-2001). Hostile environments, stereotype threat, and math performance among undergraduate women. Current Psychology : Developmental, Learning, Personality, Social, 19(4), 338-356.

Programme international pour le suivi des acquis des élèves (2001). À la hauteur : La performance des jeunes du Canada en lecture, en mathématique et en sciences. [En ligne] Disponible le 16 novembre 2010 : http://www.pisa.gc.ca/pisa/81-590xpf.pdf

Programme international pour le suivi des acquis des élèves (2003). À la hauteur : La performance des jeunes du Canada en mathématiques, en lecture, en sciences et en résolution de problèmes. [En ligne] Disponible le 16 novembre 2010 : http://www.pisa.gc.ca/81-590-xif2004001.pdf

Programme international pour le suivi des acquis des élèves (2006). À la hauteur : Résultats canadiens de l'étude PISA de I'OCDE : La performance des jeunes du Canada en sciences, en lecture et en mathématiques. [En ligne] Disponible le 16 novembre $2010:$ http://www.statcan.gc.ca/pub/81-590-x/81-590-x2007001-fra.pdf

Sekaquaptewa, D. et Thompson, M. (2003). Solo status, stereotype threat, and performance expectancies : Their effects on women's performance. Journal of Experimental Social Psychology, 39(1), 68-74.

Shih, M., Ambady, N., Richeson, J.A., Fujita, K. et Gray, H. M. (2002). Stereotype performance boosts : The impact of selfrelevance and the manner of stereotype activation. Journal of Personality and Social Psychology, 83(3), 638-647. 
Shih, M., Pittinsky, T. L. et Ambady, N. (1999). Stereotype susceptibility : Identity salience and shifts in quantitative performance. Psychological Science, 10(1), 80-83.

Smith, J. L. (2004). Understanding the process of stereotype threat : A review of mediational variables and new performance goal directions. Educational Psychology Review, 16(3), 177-206.

Steele, C. M. (1997). A threat in the air : How stereotypes shape intellectual identity and performance. American Psychologist, 52(6), 613-629.

Steele, C. M. et Aronson, J. (1995). Stereotype threat and the intellectual test performance of African Americans. Journal of Personality and Social Psychology, 69(5), 797-811.

Steele, C. M., Spencer, S. J. et Aronson, J. (2002). Contending with group image : The psychology of stereotype and social identity threat. Dans M. P. Zanna (Dir.), Advances in experimental social psychology (Vol. 34, p. 379-440). San Diego : Academic Press.

Wheeler, C. S. et Petty, R. E. (2001). The effects of stereotype activation on behavior : A review of possible mechanisms. Psychological Bulletin, 127(6), 797-826.

Yopyk, D. J. A. et Prentice, D. A. (2005). Am I an athlete or a student? Identity salience and stereotype threat in studentathletes. Basic and Applied Social Psychology, 27(4), 329-336.

Yzerbyt, V. et Schadron, G. (1994). Stéréotypes et jugement social. Dans R. Y. Bourhis et J.-P. Leyens (Dir.), Stéréotypes, discrimination et relations intergroupes (p. 127-160). Liège : Mardaga. 